TecnoLógicas

ISSN-p 0123-7799

ISSN-e 2256-5337

Vol. 23, No. 49, pp. 161-184

Sep-dic de 2020

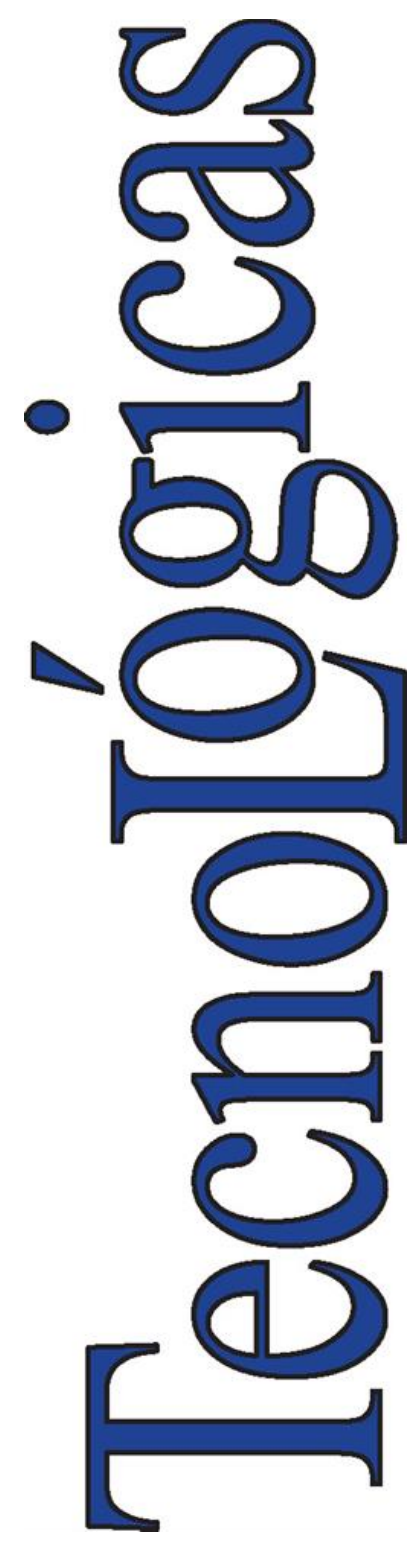

(C) Instituto Tecnológico Metropolitano Este trabajo está licenciado bajo una Licencia Internacional Creative Commons Atribución (CC BY-NC-SA)
Artículo de investigación/Research Article

\section{Nueva metodología para la evaluación de productos inhibidores de incrustación basada en modelos existentes}

\section{New Methodology for Evaluation of Scale Inhibitor Products Based on Existing Models}

\author{
Valentina Hernández-Pinto(D) 1 \\ Manuel Rodríguez-Muchati (D)2 \\ Luis Castillo-Campos $\mathbf{O}_{3}$
}

Recibido: 17 de abril de 2020

Aceptado: 06 de junio de 2020

Cómo citar / How to cite

V. Hernández-Pinto, M. Rodríguez-Muchati, L. Castillo-Campos, "Nueva metodología para la evaluación de productos inhibidores de incrustación basada en modelos existentes", TecnoLógicas, vol. 23, no. 49, pp. 161-184, 2020. https://doi.org/10.22430/22565337.1648

1 Ingeniero de Petróleo, Laboratorio de Procesamiento de Hidrocarburos, Departamento de Ingeniería de Petróleo, Escuela de Ingeniería y Ciencias Aplicadas, Universidad de Oriente-Núcleo de Monagas, Maturín-Venezuela, valentinahp96@gmail.com

2 Ingeniero de Petróleo, Laboratorio de Procesamiento de Hidrocarburos, Departamento de Ingeniería de Petróleo, Escuela de Ingeniería y Ciencias Aplicadas, Universidad de Oriente-Núcleo de Monagas, Maturín-Venezuela, mnuel1994@gmail.com

3 MSc. en Ciencias Administrativas, Laboratorio de Procesamiento de Hidrocarburos, Departamento de Ingeniería de Petróleo, Escuela de Ingeniería y Ciencias Aplicadas, Universidad de Oriente-Núcleo de Monagas, Maturín-Venezuela, lcastillo@udo.edu.ve 


\title{
Resumen
}

Las incrustaciones minerales representan un fenómeno recurrente en la industria petrolera que puede ser controlado para evitar problemas operacionales costosos. Para ello se requiere de la aplicación de tratamientos químicos conocidos como inhibidores, los cuales son evaluados tomando en cuenta propiedades como la dureza cálcica o el $\mathrm{pH}$, de manera independiente. Con base en lo expuesto, se desarrolló una metodología que permitió involucrar de forma conjunta distintos parámetros fisicoquímicos para evaluar la eficiencia de inhibidores de incrustaciones de carbonato de calcio; para efectos de esta investigación, se utilizaron dos productos comerciales y un inhibidor natural a base del mucílago extraído de la planta de cayena. En primer lugar, se realizó una esquematización de la metodología evaluativa que se quería desarrollar y que se fundamentó en la unión de tres etapas: valoración mediante la norma NACE TM 0374-2007, que toma en cuenta la dureza cálcica; el índice de saturación de Langelier para determinar la naturaleza incrustante de las aguas y un modelo de medición de $\mathrm{pH}$ continúo desarrollado por Zhang, Wu, Li y Zhao. Posteriormente, como parte de la validación de la propuesta, se caracterizaron tres muestras de salmueras sintéticas, y consecutivamente, considerando distintas dosificaciones (55, 100 y $300 \mathrm{ppm})$ de los productos inhibidores, se aplicó la metodología establecida. Se concluyó que esta nueva propuesta permite evaluar el desempeño de los inhibidores seleccionados en cada una de sus etapas, para así determinar más certeramente la capacidad antiincrustante de los mismos en muestras de salmueras sintéticas a distintas concentraciones, donde se prevea la deposición de incrustaciones minerales de carbonato de calcio $\left(\mathrm{CaCO}_{3}\right)$.

\section{Palabras clave}

Carbonato de calcio, dureza cálcica, industria petrolera, medición de $\mathrm{pH}$, salmueras sintéticas.

\begin{abstract}
Mineral scales are a current phenomenon in the oil industry that may be controlled to avoid expensive operational problems. To minimize these problems, it is necessary the application of chemical treatments known as inhibitors which are evaluated considering few parameters such as calcium hardness or $\mathrm{pH}$. As a consequence, a methodology which jointly takes into account physicochemical properties to evaluate efficiency of calcium carbonate scale inhibitors was developed; two commercial products and one organic antiscalant derived from the cayenne mucilage were used. In the first place, an evaluative methodology schematization to be developed was carried out, based on the union of three phases: assessment using the NACE Standard TM 0374-2007, which takes into account calcium hardness; the Langelier saturation index to determine the water scaling nature and a continuous $\mathrm{pH}$ measurement model developed by Zhang, $\mathrm{Wu}, \mathrm{Li}$ and Zhao. Later, to validate the proposal, three samples of synthetic brines were characterized, and consecutively, considering different dosages $(55,100$ and $300 \mathrm{ppm})$ of the inhibitor products, the established methodology was applied. It was concluded that this new proposal allows evaluating the selected inhibitors performance in each of their stages, to accurately determine their antiscaling capacity in synthetic brines samples at different concentrations, where the mineral scales deposition of calcium carbonate $\left(\mathrm{CaCO}_{3}\right)$ is expected.
\end{abstract}

\section{Keywords}

Calcium carbonate, calcium hardness, oil industry, $\mathrm{pH}$ measurement, synthetic brines. 


\section{INTRODUCCIÓN}

La industria petrolera actual se encuentra en una lucha constante por la mejora de sus operaciones, así como el aumento de la rentabilidad de las actividades que esta desarrolla. Las fluctuaciones económicas que afectan constantemente al precio del petróleo y la dificultad de explotación de las reservas nacionales remanentes han desembocado en la necesidad de llevar a cabo apropiadamente los procedimientos durante la producción, así como también, la implementación de nuevas tecnologías y técnicas que permitan el desarrollo óptimo de todo el proceso [1].

Es así como debe enfrentarse a una serie de dificultades que amenazan el desarrollo de las operaciones correspondientes a las distintas etapas de producción; entre ellas se encuentran las acumulaciones de sedimentos inorgánicos (incrustaciones) en las instalaciones implicadas, estando estos precipitados compuestos principalmente por carbonato de calcio, sulfato de bario, sulfato de estroncio, sales de magnesio, entre otros [2]. La deposición de incrustaciones indeseables generalmente causa una serie de problemas técnicos y económicos, como por ejemplo las obstrucciones de tuberías, intercambiadores de calor, equipos de superficie [2], [3], [4], [5], entre otros, que se traducen en la disminución de la tasa de flujo [6], [7] y en pérdidas millonarias para la industria [8], [9].

En los campos petroleros la mayor parte de estas incrustaciones provienen del agua de formación asociada a la extracción de los hidrocarburos; esta agua es capaz de disolver gran cantidad de compuestos minerales y es altamente rica en iones, además es sometida a diferentes cambios termodinámicos durante el proceso de producción. Aunado a esto, se considera con frecuencia su uso en métodos de recuperación secundaria, lo que podría llegar a afectar propiedades importantes como el pH y la saturación iónica de la fase acuosa por incompatibilidad en el yacimiento o en el fondo del pozo. Estas afecciones suelen traducirse en la superación del punto máximo de saturación de la fase, en el cual ya no es posible aceptar más minerales disueltos, produciendo así la formación de compuestos inorgánicos como el carbonato de calcio, el sulfato de calcio y bario [10], [11].

Es imposible prevenir la formación y el crecimiento de estas precipitaciones minerales, sin embargo, existen tratamientos químicos asociados a la aplicación de ciertos aditivos cuya función es retardar la deposición de los sedimentos en zonas críticas. Estos agentes, conocidos como inhibidores de incrustación, actúan de modo que generan un bloqueo al desarrollo de los compuestos minerales presentes en el agua de producción, impidiendo así el crecimiento de los núcleos mineralógicos [12], y pueden ser formulaciones sintéticas o productos naturales derivados de plantas como el Aloe Vera [13], la vaina de Okra [14] o el mucílago de la hoja de cayena [15].

Por otra parte, es importante destacar que la gran mayoría de los métodos para establecer la eficiencia de los inhibidores están basados en la determinación de la turbidez de la solución, el tiempo de inducción, la conductividad, así como la masa y morfología de la precipitación [16]; o en su defecto, muchas de las normas existentes solo consideran parámetros independientes y puntuales como la dureza cálcica, no tomando en cuenta la medición y relación conjunta entre propiedades como la misma dureza cálcica, el contenido de sólidos resultantes de la aplicación del producto o el $\mathrm{pH}$.

Es por ello que a lo largo de los años se han empleado y desarrollado técnicas y métodos para medir la eficiencia de inhibidores de incrustación en distintas áreas como intercambiadores de calor, procesos de desalinización de aguas, 
producción de petróleo y gas, entre otros [16], siendo los métodos más utilizados en operaciones de campo a nivel de industria petrolera, el estático de jarra [17] y el dinámico de bloqueo de tubo, descrito en la norma NACE TM 0197-97 [18] y en los estudios de Liu et al. [19], Wang et al. [20], Al Helal et al.[21], Sanni et al. [22] y Macedo et al. [23].

Entre las investigaciones relacionadas con la determinación de la eficiencia de antiincrustantes, apoyados en parámetros puntuales y que se han desarrollado en los últimos años, están los de Demadis y Lykoudis [24] y Tantayakom et al. [25] (fundamentados en la medición de la turbidez de la solución); respecto a la conductividad [26], tiempo de inducción [27], [28], [29], así como los de Gal et al. [30], Zhang et al. [31], Kiaei y Haghtalab [32], Sousa et al. [16], Lee et al [33], Suárez-Domínguez et al [34] y, GonzálezDávila y Suárez-Domínguez [35] basados en el pH de la solución; además de estudios relacionados con el uso de técnicas electroquímicas [36], [37].

En la presente investigación se tuvo como premisa investigativa desarrollar una metodología que permitiese la sinergia entre técnicas convencionales y métodos recientes, para así generar una guía que admitiera evaluar el comportamiento de cualquier inhibidor para incrustaciones de carbonato de calcio, facilitando así la selección y aplicación del producto químico que mejor se adapte al escenario presentado. Para ello, inicialmente se estableció la metodología fundamentada en el modelo de Zhang, Wu, Li y Zhao [31], Langelier [38], y la norma NACE TM03742007 [17] (basada en la determinación de dureza cálcica) y cimentada en criterios evaluativos establecidos por los investigadores. A continuación, y como parte de la validación de la propuesta metodológica, se formularon y caracterizaron tres muestras de aguas sintéticas, en las cuales fueron evaluados tres inhibidores de incrustación disponibles en el laboratorio, aplicando la metodología previamente desarrollada.

Aclarando además que el uso de muestras de salmueras sintéticas carbonatadas se realizó con la finalidad de realizar una primera validación del método en un medio con elevada inestabilidad de sus componentes salinos constituyentes y que a su vez generara un entorno con mayor tendencia incrustante.

\section{METOdologíA}

\subsection{Especificación del procedimiento realizado para la aplicación de la nueva metodología}

Las pruebas experimentales fueron realizadas en los Laboratorios de Procesamiento de Hidrocarburos, Yacimientos y Perforación de la Universidad de Oriente en el Núcleo de Monagas, Campus Los Guaritos, estado Monagas, Venezuela.

El protocolo del experimento asociado a la realización y validación de la metodología se basó en el diagrama de flujo mostrado en la Fig. 1 que consistió, como ya se ha reseñado, en la conjunción de la norma NACE TM 0374-2007 [17], la cual toma en cuenta la dureza cálcica, el índice de saturación de Langelier [38] para determinar la naturaleza incrustante o corrosiva de las aguas, y un modelo de medición de $\mathrm{pH}$ continúo desarrollado por Zhang, Wu, Li y Zhao [31].

La secuencia estructurada del procedimiento debe iniciar con:

(1) La caracterización de las muestras de aguas que se vayan a emplear como medio para medir la eficiencia de los productos antiincrustantes que se deseen evaluar, los cuales deberán ser seleccionados (3) para posteriormente establecer sus dosificaciones (4).

A continuación, las propiedades a determinar son: $\mathrm{pH}$, dureza cálcica, alcalinidad, sólidos disueltos $\mathrm{y}$ 
conductividad eléctrica. Haciendo énfasis en el hecho de que las muestras a utilizar pueden ser de aguas de producción (generadas en cualquier proceso de la industria petrolera $u$ otra industria), o como en el caso particular de este estudio, salmueras sintéticas, siempre y cuando dichos fluidos sean propensos a la generación de incrustaciones de carbonato de calcio.



Fig. 1. Nueva metodología desarrollada para evaluar el desempeño de inhibidores de incrustación Fuente: elaboración propia. 
(2) A continuación, y de manera paralela a la selección de los inhibidores, se debe proceder a la determinación del Índice de Saturación de Langelier [38] de las muestras de agua a temperatura ambiente, con la información aportada por las propiedades determinadas en la primera fase. Se utilizó el Índice de Saturación de Langelier [38] como método predictivo para verificar si las sales contenidas en las muestras de aguas formarían incrustaciones o se mantendrían disueltas; para ello se emplean (1), (2), (3), (4), (5) y (6):

$$
\begin{gathered}
\text { LSI: } \mathrm{pH}-\mathrm{pHs} \\
\mathrm{pHs}=(9,3+\mathrm{A}+\mathrm{B})-(\mathrm{C}+\mathrm{D}) \\
\mathrm{A}=-13,12 \times \log \left(\mathrm{T}^{\circ} \mathrm{C}+273\right)+34,55 \\
\mathrm{~B}=\frac{\log (\mathrm{TDS})-1}{10} \\
\mathrm{C}=\log \mathrm{Ca}^{2+}-0,4 \\
\mathrm{D}=\log \mathrm{HCO}_{3}^{-}
\end{gathered}
$$

Donde:

LSI: Índice de Saturación de Langelier. $\mathrm{pH}$ : $\mathrm{pH}$ real del agua.

pHs: $\mathrm{pH}$ al cual el agua estará saturada con $\mathrm{CaCO}_{3}$.
Para este método se definen como incrustantes, corrosivos o neutros a las muestras de aguas tal y como se establece en el método de Langelier [38] (Tabla 1).

Siendo ideales aquellas soluciones neutras o aquellas que se acerquen más a este comportamiento.

(5) Posteriormente se debería aplicar la norma NACE TM 0374-2007 [17], finalizada la misma, se requerirá de la determinación de nuevos valores de dureza cálcica de cada una de las muestras de aguas dosificadas con los productos previamente seleccionados, así como la dureza cálcica de las referidas muestras sin dosificación alguna (blanco caliente).

Además de la propiedad anteriormente citada, es necesaria una nueva caracterización (6) ( $\mathrm{pH}$, alcalinidad, sólidos disueltos y conductividad eléctrica) de las salmueras sometidas a prueba, pero esta vez bajo las condiciones obtenidas posteriores al período y temperatura especificadas por la norma $\left(71 \pm 1{ }^{\circ} \mathrm{C}\right)$.

(7) Con la data de dureza cálcica arrojada en la etapa (6) es posible determinar la eficiencia de los productos inhibidores al aplicar (7):

$$
\% \mathrm{E}=\frac{(\mathrm{Ca}-\mathrm{Cb})}{(\mathrm{Cc}-\mathrm{Cb})} \times 100
$$

\begin{tabular}{|c|c|}
\hline Caso & Interpretación \\
\hline LSI> 0 & $\begin{array}{l}\text { Se prevé la formación de incrustaciones, y pueden ocurrir precipitaciones } \\
\text { minerales }\end{array}$ \\
\hline $\mathbf{L S I}=0$ & $\begin{array}{l}\text { El agua está en estado esencialmente neutro. No se anticipa la formación de } \\
\text { incrustaciones. }\end{array}$ \\
\hline $\mathbf{L S I}<0$ & No hay potencial de incrustaciones, el agua disolverá al mineral. \\
\hline
\end{tabular}

Donde:

Ca: dureza cálcica en la muestra con tratamiento químico después de la precipitación, en ppm.

Tabla 1. Interpretación del LSI. Fuente: [38]. 
Cb: dureza cálcica en el blanco después de la precipitación, en ppm.

Cc: dureza cálcica en el blanco antes de la precipitación, en ppm.

(8) Siendo este método capaz de arrojar una eficiencia directa, se consideró el resultado obtenido y se interpretó a través de los siguientes criterios (Tabla 2), asignándose un puntaje a los resultados alcanzados que al final permitieron calificar el desempeño general del inhibidor en la matriz evaluativa.

Tabla 2. Criterios de valoración en prueba NACE TM 0374 -2007. Fuente: elaboración propia.

\begin{tabular}{ccc}
\hline Criterios & Eficiencia \% & $\begin{array}{c}\text { Puntaje para } \\
\text { calificar desempeño } \\
\text { general }\end{array}$ \\
\hline Deficient & {$[0-25)$} & 1 puntos \\
Regular & {$[25-50)$} & 4 puntos \\
Efectivo & {$[50-75)$} & 7 puntos \\
Muy & {$[75-100]$} & 10 puntos \\
\hline
\end{tabular}

(9) Con los resultados de la caracterización de las muestras de agua de la fase (6) también es posible determinar nuevamente el Índice de Saturación de Langelier [38], pero en esta oportunidad a las condiciones alcanzadas posterior a la aplicación de la norma NACE TM 03742007 [17].

(10) El cálculo del índice de saturación se aplica antes y después del suministro del tratamiento químico con la finalidad de determinar si la adición del mismo ocasiona algún cambio representativo. Asimismo, se calcula un porcentaje de variación del LSI, para determinar cuantitativamente la diferencia experimentada por el índice posterior al tiempo designado. Los resultados se obtienen a través de (8):

$$
\% \mathrm{VTI}=100-\left(\frac{\mathrm{ISM}}{\mathrm{BF}} \times 100\right)
$$

Donde:

VTI: variación de tendencia incrustante.

ISM: índice de saturación de la muestra tratada luego de las 24 horas.

BF: índice de saturación de la muestra sin tratamiento químico antes de las 24 horas.

(11) Los criterios evaluativos expuestos en la Tabla 3, cotejan la variación de tendencia incrustante antes y después de la dosificación de los antiincrustantes, determinada a través de (8).

Tabla 3. Criterios de valoración según variación de tendencia incrustante. Fuente: elaboración propia.

\begin{tabular}{ccc} 
Criterios & VTI \% & $\begin{array}{c}\text { Puntaje para calificar } \\
\text { desempeño general }\end{array}$ \\
\hline Deficiente & {$[0-10)$} & 1 puntos \\
Regular & {$[10-20)$} & 4 puntos \\
$\begin{array}{c}\text { Efectivo } \\
\text { Muy } \\
\text { efectivo }\end{array}$ & {$[20-60)$} & 7 puntos \\
\hline
\end{tabular}

(12) De manera simultánea con la etapa (4), ha de aplicarse el tercer modelo para determinación de eficiencia, que se corresponde con el desarrollado por Zhang, Wu, Li y Zhao [31]. A través de este método se observa la variación de $\mathrm{pH}$ con respecto al tiempo, tomando en consideración que el inhibidor más óptimo será el que mantenga un valor de $\mathrm{pH}$ estable, retrasando alcanzar el umbral de precipitación mineral. Para ello se registran valores de $\mathrm{pH}$ cada minuto, culminando el experimento una vez que ocurra la precipitación mineral junto con una disminución del $\mathrm{pH}$.

(13) Posterior a ello, se procede a calcular el índice de supersaturación (S), el cual se asocia al momento exacto en el cual ocurra la formación de la incrustación, como se expresa en (9):

$$
\mathrm{S}=\frac{\left[\mathrm{Ca}^{2+}\right]\left[\mathrm{CO}_{3}{ }^{2-}\right]}{\mathrm{K}_{\mathrm{sp}}}
$$


Donde:

$\left[\mathrm{Ca}^{2+}\right]$ : concentración de iones de calcio, ppm.

$\left[\mathrm{CO}_{3}{ }^{2-}\right]:$ concentración de iones de carbonato, ppm.

Ksp: constante de disociación del carbonato de calcio, adimensional.

(14) Para efectos de esta etapa, se calcula la variación del índice de supersaturación (10) para cuantificar el cambio una vez alcanzado el umbral de precipitación.

Se toma como referencia máxima la muestra de agua con dosificación química que presenta el índice de supersaturación más alto para cada una de las aguas, debido a que estas retrasaron la formación de la incrustación por más tiempo.

$$
\% \text { VIS }=\left(\frac{S}{\operatorname{SDM}} \times 100\right)-100
$$

Donde:

VIS: variación del índice de supersaturación.

S: índice de supersaturación del ejemplar estudiado de muestra de agua (con o sin tratamiento químico).

SDM: índice de supersaturación máximo en la misma muestra de agua.

(15) Para determinar la eficiencia de los químicos se estableció el siguiente criterio de valoración definido en la Tabla 4.

Tabla 4. Criterio de valoración según variación de índice de supersaturación Fuente: elaboración propia.

\begin{tabular}{ccc}
\hline Criterios & VIS, \% & $\begin{array}{c}\text { Puntaje para calificar } \\
\text { desempeño general }\end{array}$ \\
\hline Deficiente & {$[0-25)$} & 1 puntos \\
Regular & {$[25-50)$} & 4 puntos \\
Efectivo & {$[50-75)$} & 7 puntos \\
Muy efectivo & {$[75-100]$} & 10 puntos \\
\hline
\end{tabular}

(16) Una vez aplicados los tres procedimientos que conforman la metodología con sus respectivos criterios evaluativos, a los inhibidores tasados en las muestras de aguas, se deben contabilizar las puntuaciones obtenidas por cada producto en los tres métodos empleados. El criterio final de valoración calificará el desempeño general del químico, describiéndolo como un inhibidor deficiente, regular, efectivo o muy efectivo.

Este desempeño general se encuentra establecido de acuerdo con los siguientes rangos definidos en la Tabla 5:

Tabla 5. Criterio de valoración para calificar el desempeño general de inhibidores Fuente: elaboración propia.

\begin{tabular}{cc}
\hline $\begin{array}{c}\text { Desempeño general del } \\
\text { inhibidor }\end{array}$ & $\begin{array}{c}\text { Puntaje } \\
\text { acumulado }\end{array}$ \\
\hline Deficiente & {$[0-7,50)$} \\
Regular & {$[7,50-15)$} \\
Efectivo & {$[15-22,50)$} \\
Muy efectivo & {$[22,50-30]$} \\
\hline
\end{tabular}

\subsection{Evaluación de los inhibidores de incrustación mediante la aplicación de la metodología establecida}

Para efectos de evaluar la metodología previamente constituida, y así validarla, hacer los ajustes respectivos y sugerir algunos cambios futuros, se hizo necesaria la selección de inhibidores de incrustación y de muestras de agua. En ambos casos, el muestreo realizado fue por conveniencia o discrecional, toda vez que tanto los componentes de las salmueras sintéticas, como los productos químicos fueron elegidos por la accesibilidad a ellos por parte de los investigadores.

Respecto a los antiincrustantes, se seleccionaron dos productos químicos comerciales provenientes de dos empresas de servicios petroleros, y un químico orgánico desarrollado por Reyes et al. [15], disponibles todos en el Laboratorio de Procesamiento de Hidrocarburos de la Universidad de Oriente, Núcleo de Monagas, Venezuela. Asociado a esta investigación y con la finalidad de preservar la confidencialidad de la información de las casas químicas, referente a los dos agentes comerciales, se les denominaron como Q1 y Q2, 
respectivamente y al aditivo orgánico como Q3.

Resulta imperioso especificar que el producto Q1 es una mezcla de alcohol metílico, sal de ácido fosfónico y ácido acético, con una reactividad de cero (0) lo que indica que es estable, de acuerdo con las consideraciones de la NFPA 704 [39], además de presentar una gravedad específica entre 1,0336 y 1,0586 y un $\mathrm{pH}$ entre 5,14 y 6,14 [40]. Mientras que Q2 es un polímero de acrilato empleado como inhibidor de carbonatos de calcio y magnesio, considerado como una mezcla no reactiva, $100 \%$ soluble en agua, con una gravedad específica $\left(\mathrm{a} 25^{\circ} \mathrm{C}\right)$ entre $1,100 \mathrm{y}$ 1,160 y un pH (a $\left.25^{\circ} \mathrm{C}\right)$ entre 5 y 7 .

En relación con el inhibidor orgánico (Q3), es un producto formulado a base de un mucílago proveniente de la hoja de cayena, compuesto por polisacáridos naturales ácidos parcialmente acetilados, ácido D-galacturónico, ácido D-glucurónico, presencia de calcio, entre otros y un $\mathrm{pH}$ de 4,17 [15].

Para el caso de las muestras de salmueras sintéticas formuladas (tres), fueron preparadas con agua destilada, y reactivos de grado analítico $\left(\mathrm{CaCl}_{2}, \mathrm{NaCl}\right.$ y $\mathrm{NaHCO}_{3}$ ) de la empresa ECCO Chemical, C.A. Destacando que se variaron las concentraciones tanto de bicarbonato de sodio $\left(\mathrm{NaHCO}_{3}\right)$ como de cloruro de calcio $\left(\mathrm{CaCl}_{2}\right)$, manteniendo las proporciones añadidas de cloruro de sodio $(\mathrm{NaCl})$, como se expone en la Tabla 6; de esta forma fue posible observar el desempeño de los inhibidores a distintas concentraciones salinas en cada una de las etapas de la metodología.

Tabla 6. Composición de muestras de agua sintética Fuente: elaboración propia.

\begin{tabular}{clll}
\hline Muestra & $\begin{array}{l}\mathrm{CaCl}_{2} \\
(\mathrm{~g} / \mathrm{L})\end{array}$ & $\begin{array}{l}\mathrm{NaHCO}_{3} \\
(\mathrm{~g} / \mathrm{L})\end{array}$ & $\begin{array}{l}\mathrm{NaCl} \\
(\mathrm{g} / \mathrm{L})\end{array}$ \\
\hline 1 & 3,33 & 2,52 & 33 \\
\hline 2 & 6,33 & 2,52 & 33 \\
\hline 3 & 2,33 & 5,52 & 33 \\
\hline
\end{tabular}

$\mathrm{Al}$ ser una metodología en etapa inicial, se usaron aguas sintéticas con la finalidad de controlar de alguna forma los constituyentes de ellas, para así medir las eficiencias de los agentes químicos en un medio relativamente ideal, a diferencia de lo que habría ocurrido al emplear aguas de producción, las cuales son mezclas más complejas de sales (solubles e insolubles), metales, hidrocarburos, sólidos inertes de los yacimientos productores, entre otros.

Las salmueras sintéticas no presentaron un grado de inestabilidad en cuanto a la precipitación temprana de la incrustación objetivo (carbonato de calcio), debido a que, para efectos de su formulación, los componentes salinos eran disueltos por separado en el agua destilada y al momento de realizar las pruebas valorativas de la metodología, se realizaba la unión de ellas, esto en clara alusión y semejanza con lo estipulado en la norma NACE TM 0374 - 2007 [17].

En resumen, las tres salmueras sintéticas y los tres inhibidores (a tres dosificaciones distintas: 55, 100 y 300 ppm) conformaron la fuente de validación del proceso evaluativo, esto con la finalidad de tener una matriz estadística representativa con tres aditivos químicos a sus tres dosis respectivas evaluados en tres medios salinos y realizando tres repeticiones para cada caso, lo que hizo un total de 81 pruebas experimentales, en cada una de las tres fases de la nueva metodología.

Posterior a la formulación, se caracterizaron las salmueras mediante la obtención de las siguientes propiedades fisicoquímicas: $\mathrm{pH}$ [41] mediante un pHmetro digital marca Denver Instrument, modelo 220; contenido de sólidos disueltos [42] con la ayuda de un horno de laboratorio marca CARBOLITEÒ y una balanza digital marca Mettler Toledo; además de conductividad eléctrica [43] (obtenida a través de un conductímetro marca ORION 3 STAR). 
Adicionalmente, se obtuvieron mediante titulación, propiedades como: alcalinidad [44], dureza cálcica [45] y cloruros [46].

\subsubsection{Determinación del índice de Saturación de Langelier (LSI)}

Con la caracterización previamente realizada a las muestras de aguas sintéticas a temperatura ambiente, la aplicación de esta fase, simplemente consistió en una determinación matemática del índice, con el uso de (1) a (6), donde son empleados parámetros como $\mathrm{pH}$, temperatura de prueba, sólidos disueltos, ión calcio (determinado con el valor de dureza cálcica) e ión bicarbonato (obtenido mediante el resultado de alcalinidad), para de esta forma corroborar la tendencia incrustante o no de los medios salinos. Ahora bien, de acuerdo con la metodología, el LSI también se determinó posterior a la aplicación de la norma NACE TM 0374 - 2007, con la finalidad de verificar si la aplicación de los productos químicos y la elevación de la temperatura, permitieron variar la propensión incrustante de las aguas.

\subsubsection{Determinación de la eficiencia a través de la normativa NACE TM 0374 - 2007}

Para aplicar la norma NACE TM 0374 - 2007 [17], las muestras de aguas sintéticas fueron colocadas en celdas de vidrio limpias de $100 \mathrm{~mL}$, marca KIMAXÒ.

Posteriormente, se adicionaron las dosis previamente establecidas (55, 100 y 300 ppm) de los inhibidores a ser evaluados, dejando una celda sin dosificación, a la cual se le denominó como blanco. Las celdas fueron dispuestas en un baño termostático marca Thermo Scientific ${ }^{\mathrm{TM}}$, modelo 18202 a $71 \pm 1{ }^{\circ} \mathrm{C}$ durante 24 horas, tal como se visualiza en la Fig. 2. Posterior al tiempo establecido, fueron extraídas del mismo, evitando ser agitadas, y se dejaron reposar hasta la temperatura ambiente, determinando nuevamente la dureza cálcica de las muestras analizadas y con ello el porcentaje de eficiencia de los inhibidores durante esta etapa a través de (7).

\subsubsection{Determinación de la eficiencia a través del modelo de medición de pH continúo desarrollado por Zhang, Wu, Li y Zhao}

El montaje experimental (Fig. 3) estuvo comprendido por una plancha de calentamiento (hot plate) marca Magnestir Lab-Line, una bureta de $250 \mathrm{~mL}$ marca Cole Parmer, un beaker de $500 \mathrm{~mL}$ con agitador magnético y un pHmetro digital marca Denver Instrument, modelo 220.

La prueba consistió en tomar una cantidad en $\mathrm{mL}$ de solución de cloruro de calcio $\left(\mathrm{CaCl}_{2}\right)$ en concentraciones de 6,66; 12,66 y $4,66 \mathrm{~g} / \mathrm{L}$, correspondientes a las tres muestras respectivas ya mencionadas; obteniéndose estas concentraciones a partir de una relación entre la cantidad de sales utilizada en los métodos anteriores y la empleada en el modelo de medición de pH continúo desarrollado por Zhang, Wu, Li y Zhao [31]. Cada una de ellas fue mezclada con una cantidad de agua destilada conforme con la proporción de $\mathrm{CaCl}_{2}$ añadida y cada una de las dosis ya estipuladas de los inhibidores elegidos para esta evaluación. Luego se tituló con bicarbonato de sodio $\left(\mathrm{NaHCO}_{3}\right)$ en concentraciones de 5 y $2,28 \mathrm{~g} / \mathrm{L}$, agregando $2 \mathrm{~mL}$ cada 60 segundos.

Los valores de $\mathrm{pH}$ fueron registrados antes de iniciar la titulación con $\mathrm{NaHCO}_{3}$ y luego de transcurridos cada 60 segundos.

El experimento terminó una vez ocurrida la precipitación de la incrustación de la mano de una disminución apreciable del

$\mathrm{pH}$. 


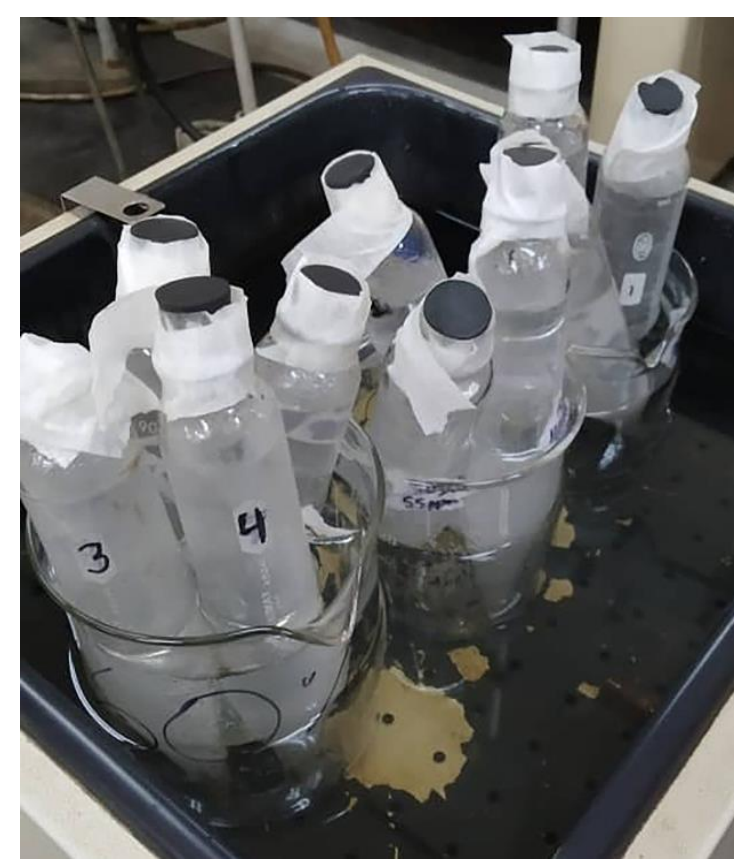

Fig. 2. Montaje experimental de la prueba de eficiencia mediante la norma NACE TM 0374 - 2007 [17] Fuente: elaboración propia.

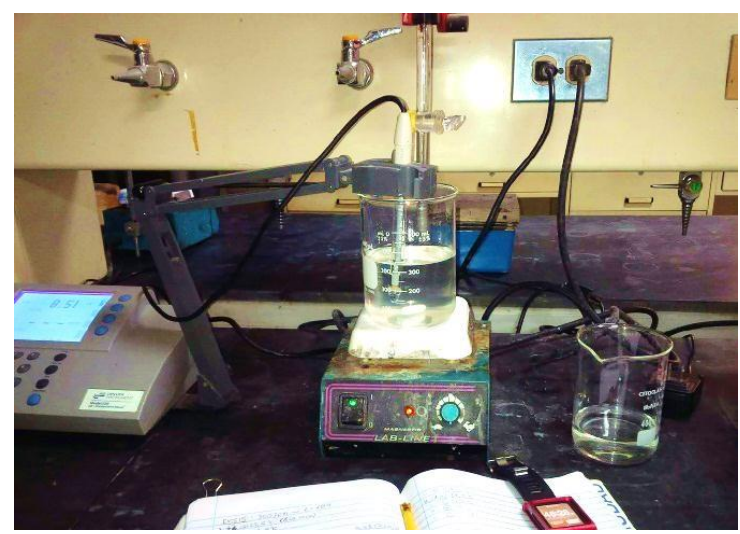

Fig. 3. Montaje experimental de la prueba de eficiencia mediante el modelo de medición de pH continuo desarrollado por Zhang et al. [31]. Fuente: elaboración propia.

\section{RESULTADOS Y DISCUSIÓN}

Con la finalidad de ratificar o permitir sugerir modificaciones a la secuencia planteada para la metodología ya señalada, los inhibidores Q1, Q2 y Q3 fueron evaluados tomando en cuenta la variación de las propiedades físicas y químicas de las muestras de agua formuladas, en cada una de las etapas que conforman esta nueva herramienta, la cual se encuentra especificada en el diagrama de flujo ilustrado en la Fig. 1 y que guió la sucesión de trabajo de esta investigación.

Acotando además que, para efectos de esta disertación, los resultados y discusión están basados en la aplicación de esta propuesta, únicamente en los fluidos utilizados, pero aclarando que la metodología no es exclusiva y limitante para ellos.

Antes de aplicar la propuesta, la totalidad de las muestras sintéticas fueron caracterizadas, presentando un alto 
carácter incrustante (ver Tabla 7), esto es evidenciable a través de los resultados obtenidos en propiedades como el $\mathrm{pH}$ donde se observan valores superiores a 7 , lo que caracteriza a las aguas como básicas o alcalinas; esta condición propicia la deposición de las sales debido a que a medida que aumenta el $\mathrm{pH}$, la solubilidad de las mismas disminuye [47].

Respecto a la alcalinidad, las muestras de aguas sintéticas estudiadas mostraron resultados entre $1000 \mathrm{mg} / \mathrm{L}$ hasta más de $3000 \mathrm{mg} / \mathrm{L}$, que de acuerdo con Porras et al. [48], se considera que alcalinidades mayores a 300 ppm de $\mathrm{CaCO}_{3}$ son catalogadas como elevadas. Dichos resultados se deben a la variación de la concentración del ion bicarbonato, el cual es un factor principal en conjunto con el ion calcio para propiciar la reacción química del carbonato de calcio [48]. Esta tendencia incrustante se ratifica con los valores de dureza cálcica obtenidos, los cuales se deben a las cantidades de sales añadidas en cada una de las muestras, que al ser todos superiores a 300 ppm, describen a la totalidad de las salmueras como "muy duras" [49] lo cual contribuye a la formación de incrustaciones.

Los resultados de sólidos disueltos obtenidos están comprendidos en un rango desde los 86.685 ppm hasta 97.690 ppm, debido a las concentraciones de $\mathrm{CaCl}_{2}$,
$\mathrm{NaHCO}_{3}$ y $\mathrm{NaCl}$ añadidas al momento de formular las aguas sintéticas. Estos valores son considerados altos si se contrastan con lo estipulado por el reglamento secundario EPA [50], que establece que el nivel de contaminación máximo recomendable por sólidos disueltos totales en aguas es de 500 ppm.

Igualmente, expresan que cuando los niveles de sólidos disueltos exceden los $1.000 \mathrm{mg} / \mathrm{L}$, el agua no es apta para procesos industriales debido a la alta presencia de sales como potasio, cloruros y sodio, los cuales pueden ocasionar problemas operacionales como acumulación de sarro en las tuberías o precipitaciones minerales.

La conductividad en las muestras de agua estudiadas fueron $343 \mu \mathrm{S} / \mathrm{cm}, 590$ $\mu \mathrm{S} / \mathrm{cm}$ y $478 \mu \mathrm{S} / \mathrm{cm}$ para las muestras 1,2 y 3 , respectivamente; indicando que la conductividad será más alta en aquellas muestras que presenten altas concentraciones salinas, como se presenta en la muestra 2. Dichos valores indican que, además de ser aguas altamente conductivas, de acuerdo con el reglamento secundario EPA [50], aquellas aguas que presenten valores entre $300 \mu \mathrm{S} / \mathrm{cm}$ y 600 $\mu \mathrm{S} / \mathrm{cm}$ se consideran altamente duras, concordando así con los valores de dureza obtenidos.

Tabla 7. Propiedades físicas y químicas de muestras de agua 1, 2 y 3. Fuente: elaboración propia.

\begin{tabular}{|c|c|c|c|}
\hline Propiedad & $\begin{array}{c}\text { Muestra } 1\left(3,33 \mathrm{CaCl}_{2}\right. \\
\left.+2,52 \mathrm{NaHCO}_{3}\right)\end{array}$ & $\begin{array}{c}\text { Muestra } 2\left(6,33 \mathrm{CaCl}_{2}\right. \\
\left.+2,52 \mathrm{NaHCO}_{3}\right)\end{array}$ & $\begin{array}{c}\text { Muestra } 3\left(2,33 \mathrm{CaCl}_{2}\right. \\
\left.+5,52 \mathrm{NaHCO}_{3}\right)\end{array}$ \\
\hline $\mathrm{pH}$ a $27^{\circ} \mathrm{C}( \pm 0,002)$ & 7,34 & 7,20 & 7,08 \\
\hline Alcalinidad parcial $(\mathrm{mg} / \mathrm{L})$ & 0 & 0 & 0 \\
\hline Alcalinidad total $(\mathrm{mg} / \mathrm{L})( \pm 1,00)$ & 1.420 & 1.250 & 3.150 \\
\hline Sólidos disueltos $(\mathrm{ppm})( \pm 0,55)$ & 86.685 & 87.400 & 97.690 \\
\hline Cloruros $(\mathrm{mg} / \mathrm{L})( \pm 1,00)$ & $1.529,5$ & $1.599,5$ & 3.410 \\
\hline Dureza cálcica $(\mathrm{mg} / \mathrm{L})( \pm 1,00)$ & 1.150 & 2.120 & 760 \\
\hline $\begin{array}{l}\text { Conductividad eléctrica }(\mu \mathrm{S} / \mathrm{cm}) \\
( \pm 0,05)\end{array}$ & 343 & 590 & 468 \\
\hline $\begin{array}{l}\text { Índice de Saturación de } \\
\text { Langelier- LSI (Adim.) }( \pm 0,01)\end{array}$ & 1,302 & 1,3241 & 0,8347 \\
\hline
\end{tabular}


Los índices de Saturación de Langelier resultaron positivos para las tres salmueras, oscilando entre $0,8347 \quad \mathrm{y}$ 1,3241, estimando así una tendencia incrustante para todas, y asociando el valor mayor a la salmuera con más elevado contenido de cloruro de calcio (muestra 2), que a su vez resultó con mayor dureza cálcica, siendo esta la que se caracteriza con mayor potencial incrustante.

\subsection{NACE TM $0374-2007$}

$\mathrm{Al}$ iniciar la aplicación de la metodología en las muestras en estudio, se evidenció que las salmueras sintéticas dosificadas con los químicos antiincrustantes presentaron cambios en las propiedades físicas y químicas posteriores a la aplicación de esta primera etapa descrita por la norma NACE TM0374 - 2007 [17]. En este sentido, se demuestra una disminución considerable de la dureza cálcica en las muestras sin dosificación química (blanco caliente) con respecto a la muestra sin dosificación química antes del período de tiempo designado por la prueba (blanco frío), punto mínimo de deposición mineral; esto se traduce en una precipitación de las sales presentes debido a las condiciones de temperatura y tiempo a las que fueron expuestas. Asimismo, la Tabla 8 indica este mismo comportamiento para las muestras de agua dosificadas a $300 \mathrm{ppm}$ de cada producto, sin embargo, la disminución no estuvo tan marcada por el efecto inhibitorio de los químicos.

Tabla 8. Dureza cálcica de muestras de agua posterior a la aplicación de la norma NACE TM0374 -2007 . Fuente: elaboración propia.

\begin{tabular}{|c|c|c|c|c|}
\hline Dosis & Inhibidor & $\begin{array}{c}\text { Muestra } \\
1 \text { (ppm) }\end{array}$ & $\begin{array}{c}\text { Muestra } \\
2 \text { (ppm) }\end{array}$ & $\begin{array}{c}\text { Muestra } \\
3 \text { (ppm) }\end{array}$ \\
\hline \multirow{3}{*}{$\begin{array}{c}300 \\
\mathrm{ppm}\end{array}$} & $\mathrm{Q}_{1}$ & 1.080 & 2.060 & 680 \\
\hline & $\mathrm{Q}_{2}$ & 1.140 & 1.860 & 560 \\
\hline & $\mathrm{Q}_{3}$ & 760 & 1.760 & 400 \\
\hline \multicolumn{2}{|c|}{ Blanco caliente } & 600 & 1.680 & 80 \\
\hline \multicolumn{2}{|c|}{ Blanco frío } & 1.150 & 2.120 & 760 \\
\hline
\end{tabular}

Por otra parte, los resultados expresados en la Tabla 9 indican un incremento en la alcalinidad total en algunas de las muestras de agua con los tres inhibidores utilizados. Esto se debe a la ocurrencia de una perturbación en el ion bicarbonato que, al verse expuesto a una temperatura de $71{ }^{\circ} \mathrm{C}$, supera su límite de disolución, interactuando así con los iones calcio presentes, dando como origen la precipitación de carbonato de calcio [51]. Este comportamiento también se evidenció en las dosis de 55 y 100 ppm en cada una de las muestras de agua formuladas.

Tabla 9. Alcalinidad total de las salmueras posterior a la NACE TM $0374-2007$ Fuente: elaboración propia.

\begin{tabular}{ccccc}
\hline Dosis & Inhibidor & Muestra & Muestra & Muestra \\
\hline \multirow{2}{*}{300} & $\mathrm{Q}_{1}$ & 1.625 & 1.438 & 3.250 \\
\cline { 2 - 5 } ppm & $\mathrm{Q}_{2}$ & 1.875 & 1.750 & 2.813 \\
\cline { 2 - 5 } & $\mathrm{Q}_{3}$ & 1.000 & 875 & 2.250 \\
\hline \multicolumn{2}{c}{ Blanco caliente } & 500 & 1.000 & 1.500 \\
\hline
\end{tabular}

Los resultados de eficiencia obtenidos a través de la norma NACE TM 0374 - 2007 [17] indican que el rendimiento de los químicos es proporcional a la dosis añadida. Partiendo de esto y de acuerdo con los resultados anexos en la Tabla 10, tanto el inhibidor Q1 como Q2 se califican como químicos muy efectivos al presentar un rendimiento del $100 \%$ y $98,18 \%$, respectivamente, en sus máximas dosis.

Caso contrario se observa en el inhibidor Q3, el cual obtuvo valores que van desde 0 hasta $35,71 \%$, este último en su dosis más alta, catalogándose como un químico inhibidor deficiente hasta regular para todas las muestras de aguas evaluadas. Dicho resultado de eficiencia se asemeja con el obtenido por Reyes et al. [15] en su estudio, el cual presentó un máximo valor de $36,73 \%$ luego de utilizar una dosificación de 500 ppm.

Asimismo, Q2 y Q3 presentan el desempeño más bajo en la muestra de agua 
Nueva metodología para la evaluación de productos inhibidores de incrustación basada en modelos existentes

2 con respecto a sus semejantes en el resto de las muestras, indicando así que el comportamiento de los inhibidores se ve afectado por la dureza cálcica que presenten las aguas [52], propiedad que se encuentra altamente relacionada con la concentración de cloruro de calcio presente.

\section{2 Índice de Saturación de Langelier (LSI)}

Seguidamente, se determinó el índice de saturación de Langelier posterior a las 24 horas de prueba. Los resultados demostraron que la totalidad de las muestras de agua se caracterizaban por ser soluciones en las que se prevé la formación de incrustaciones al presentar índices de saturación superiores a cero (0) (Tabla 11).

Tabla 10. Resultados de eficiencia posterior a la norma NACE TM $0374-2007$

Fuente: elaboración propia.

\begin{tabular}{|c|c|c|c|}
\hline Muestra & Inhibidor & Dosis, ppm & Eficiencia, \% $( \pm 0,01)$ \\
\hline \multirow{9}{*}{$\begin{array}{c}1\left(3,33 \mathrm{~g} \mathrm{CaCl}_{2}\right. \\
\left.+2,52 \mathrm{~g} \mathrm{NaHCO}_{3}\right)\end{array}$} & \multirow{3}{*}{ Q1 } & 55 & 72,72 \\
\hline & & 100 & 83,63 \\
\hline & & 300 & 87,27 \\
\hline & \multirow{3}{*}{$\mathrm{Q} 2$} & 55 & 83,63 \\
\hline & & 100 & 87,27 \\
\hline & & 300 & 98,18 \\
\hline & \multirow{3}{*}{ Q3 } & 55 & 11,76 \\
\hline & & 100 & 17,65 \\
\hline & & 300 & 29,41 \\
\hline \multirow{9}{*}{$\begin{array}{l}2\left(6,33 \mathrm{~g} \mathrm{CaCl}_{2}+\right. \\
\left.2,52 \mathrm{~g} \mathrm{NaHCO}_{3}\right)\end{array}$} & \multirow{3}{*}{ Q1 } & 55 & 54,54 \\
\hline & & 100 & 86,36 \\
\hline & & 300 & 100 \\
\hline & \multirow{3}{*}{ Q2 } & 55 & 13,64 \\
\hline & & 100 & 54,54 \\
\hline & & 300 & 40,91 \\
\hline & \multirow{3}{*}{ Q3 } & 55 & 2,27 \\
\hline & & 100 & 6,82 \\
\hline & & 300 & 18,18 \\
\hline \multirow{9}{*}{$\begin{array}{c}3\left(2,33 \mathrm{~g} \mathrm{CaCl}_{2}\right. \\
\left.+5,52 \mathrm{~g} \mathrm{NaHCO}_{3}\right)\end{array}$} & \multirow{3}{*}{ Q1 } & 55 & 76,47 \\
\hline & & 100 & 82,35 \\
\hline & & 300 & 88,26 \\
\hline & \multirow{3}{*}{ Q2 } & 55 & 47,06 \\
\hline & & 100 & 79,41 \\
\hline & & 300 & 70,59 \\
\hline & \multirow{3}{*}{ Q3 } & 55 & 0 \\
\hline & & 100 & 0 \\
\hline & & 300 & 35,71 \\
\hline
\end{tabular}


Nueva metodología para la evaluación de productos inhibidores de incrustación basada en modelos existentes

Tabla 11. Variación de la tendencia incrustante de las muestras de agua, de acuerdo con el LSI Fuente: elaboración propia.

\begin{tabular}{|c|c|c|c|c|}
\hline Muestra & Inhibidor & Dosis, ppm & LSI $( \pm 0,01)$ & Variación de tendencia incrustante, \% \\
\hline \multirow{10}{*}{$\begin{array}{c}1\left(3,33 \mathrm{~g} \mathrm{CaCl}_{2}+2,52\right. \\
\left.\mathrm{g} \mathrm{NaHCO}_{3}\right)\end{array}$} & & 0 & 1,302 & \\
\hline & \multirow{3}{*}{ Q1 } & 55 & 1,0003 & 23,17 \\
\hline & & 100 & 1,0505 & 19,32 \\
\hline & & 300 & 1,0208 & 21,60 \\
\hline & \multirow{3}{*}{ Q2 } & 55 & 1,1018 & 15,38 \\
\hline & & 100 & 1,1151 & 14,35 \\
\hline & & 300 & 1,1476 & 11,86 \\
\hline & \multirow{3}{*}{ Q3 } & 55 & 1,2448 & 4,39 \\
\hline & & 100 & 1,2674 & 2,66 \\
\hline & & 300 & 1,3448 & $-3,29$ \\
\hline \multirow{10}{*}{$\begin{array}{c}2\left(6,33 \mathrm{~g} \mathrm{CaCl}_{2}+2,52\right. \\
\left.\mathrm{g} \mathrm{NaHCO}_{3}\right)\end{array}$} & & 0 & 1,3241 & \\
\hline & \multirow{3}{*}{ Q1 } & 55 & 1,1227 & 15,21 \\
\hline & & 100 & 1,1549 & 12,78 \\
\hline & & 300 & 1,2409 & 6,28 \\
\hline & \multirow{3}{*}{ Q2 } & 55 & 1,0771 & 18,65 \\
\hline & & 100 & 1,1422 & 13,74 \\
\hline & & 300 & 1,1917 & 10 \\
\hline & \multirow{3}{*}{ Q3 } & 55 & 1,3124 & 0,89 \\
\hline & & 100 & 1,3924 & $-5,16$ \\
\hline & & 300 & 1,3701 & $-3,47$ \\
\hline \multirow{10}{*}{$\begin{array}{c}3\left(2,33 \mathrm{~g} \mathrm{CaCl}_{2}+5,52\right. \\
\left.\mathrm{g} \mathrm{NaHCO}_{3}\right)\end{array}$} & & 0 & 0,8347 & \\
\hline & \multirow{3}{*}{ Q1 } & 55 & 0,7501 & 10,13 \\
\hline & & 100 & 0,7434 & 10,93 \\
\hline & & 300 & 0,8101 & 2,94 \\
\hline & \multirow{3}{*}{ Q2 } & 55 & 0,4596 & 44,94 \\
\hline & & 100 & 0,7385 & 11,53 \\
\hline & & 300 & 0,6429 & 22,98 \\
\hline & \multirow{3}{*}{ Q3 } & 55 & 1,2562 & $-50,50$ \\
\hline & & 100 & 1,3096 & $-56,89$ \\
\hline & & 300 & 0,9083 & $-8,82$ \\
\hline
\end{tabular}

Es importante destacar que, aunque todas las muestras presentan una clara tendencia incrustante, aquellas tratadas con los inhibidores Q1 y Q2 presentaron índices más cercanos al punto neutro, es decir, a un LSI=0, lo que indica que los químicos lograron neutralizar dicha condición incrustante. Caso contrario se observó en las muestras tratadas con el inhibidor Q3, donde algunas soluciones llegaron incluso a superar el punto máximo incrustante correspondiente a la muestra de agua sin dosificación alguna (blanco caliente), dando como conclusión que el químico contribuye a la formación de incrustaciones.

La discordancia de resultados entre las muestras de agua con distintas dosificaciones se debe a las concentraciones de sal presentes en la mismas, más específicamente a la concentración del cloruro de calcio. Al tratarse de una de las 
sales predominantes en la formación del carbonato de calcio, es correcto afirmar que mientras menor sea la concentración de la misma, las muestras de agua van a presentar un índice de saturación más cercano al punto neutro [53].

Tomando en cuenta de que esta etapa se basa en la utilización de un método predictivo, para lograr cuantificar la eficiencia de los inhibidores se determinó la variación de la tendencia incrustante de las aguas comparándola con la muestra sin dosificación química; en este caso, aquellos porcentajes más altos indicaban una mayor diferencia con respecto a este valor, denotando así mayor efectividad del tratamiento, por ende, aquellos resultados bajos o negativos corresponden a tratamientos regulares y deficientes.

En este sentido, el inhibidor Q1 obtuvo un desempeño catalogado como efectivo tanto para la muestra 1 como para la 3 , así mismo, este se vio reducido para la muestra 2 al presentar la mayor concentración cálcica.

Respecto al inhibidor Q2, se observa un desempeño bajo, tanto para la muestra 1 como para la 2, describiéndolo como un tratamiento que va desde regular hasta deficiente. Sin embargo, en la muestra 3 , se observan resultados más satisfactorios que denotan un comportamiento que va desde efectivo a muy efectivo; razón que está relacionada a la poca concentración cálcica presente en la muestra de agua.

Reyes et al. [15] evaluaron el comportamiento del inhibidor Q3 y evidenciaron la elevada dureza cálcica que presenta el mismo; dicha afirmación se confirma con los resultados obtenidos en las distintas muestras que lo describen como un inhibidor regular y deficiente al presentar porcentajes de variación bajos y negativos.

\subsection{Modelo de medición de pH continuo desarrollado por Zhang, Wu, Li y Zhao}

A continuación, las muestras de aguas sintéticas fueron sometidas a una medición de $\mathrm{pH}$ continuo, basándose en el modelo desarrollado por Zhang et al. [31], de los cuales se obtuvo un comportamiento para la muestra de agua 1 que fue ilustrado en la Fig. 4. El inhibidor Q1 fue capaz de retrasar la llegada al umbral de precipitación hasta 137 minutos; punto en el cual ocurre la precipitación máxima y, por ende, el pH de la muestra disminuye, deduciendo que, en presencia del inhibidor, el sistema se mantiene en una condición sobresaturada más alta, en comparación con el experimento no inhibido; comportamientos que fueron replicados en los estudios de Sousa et al. [16], 75 minutos para el mejor inhibidor) y Zhang et al. [31] (Fig. 5).

Así mismo, se observa como en todas las dosificaciones del químico ocurrió una repetición o bucle de los valores de $\mathrm{pH}$ que se prolongó durante determinado tiempo y estuvo asociado a las bajas concentraciones salinas en comparación con las utilizadas por Zhang et al. [31], corroborando que aparte del $\mathrm{pH}$, las concentraciones de calcio y bicarbonato son parámetros fundamentales en el control de las incrustaciones en cualquier muestra de agua (sea sintética o no).

La diferencia existente entre las concentraciones salinas de las muestras de agua estudiadas se visualiza al comparar el comportamiento del $\mathrm{pH}$ presentado en la Fig. 5 con la Fig. 4, donde los autores originales utilizaron $34 \mathrm{~mL}$ de bicarbonato de sodio $\left(\mathrm{NaHCO}_{3}\right)$, agregando $1 \mathrm{~mL}$ cada 1 minuto transcurrido, llegando a retrasar el umbral de precipitación hasta 34 minutos. 
Nueva metodología para la evaluación de productos inhibidores de incrustación basada en modelos existentes

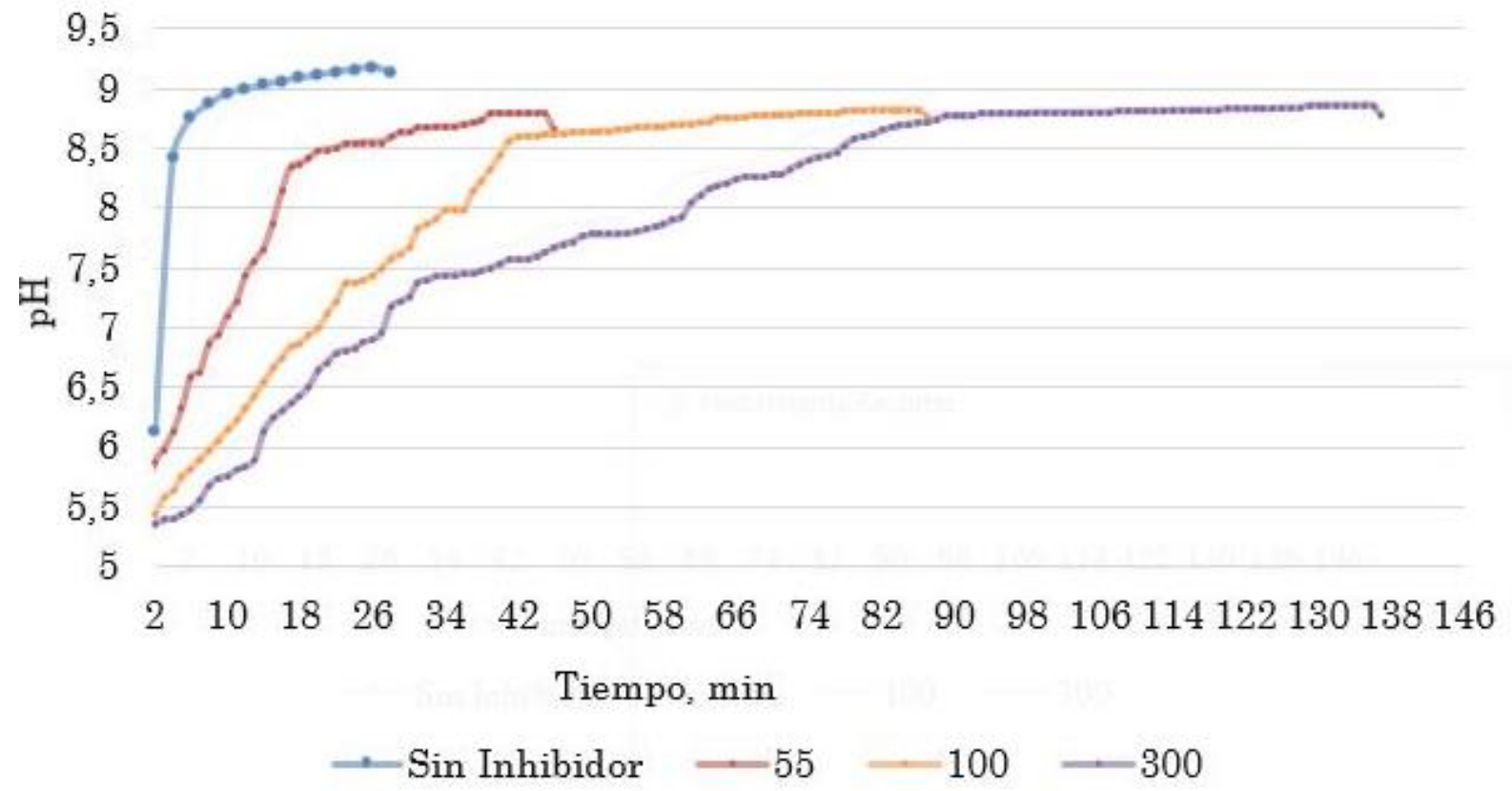

Fig. 4. Comportamiento de $\mathrm{pH}$ en muestra de agua sintética 1 tratada con inhibidor $\mathrm{Q}_{1}$ Fuente: elaboración propia.

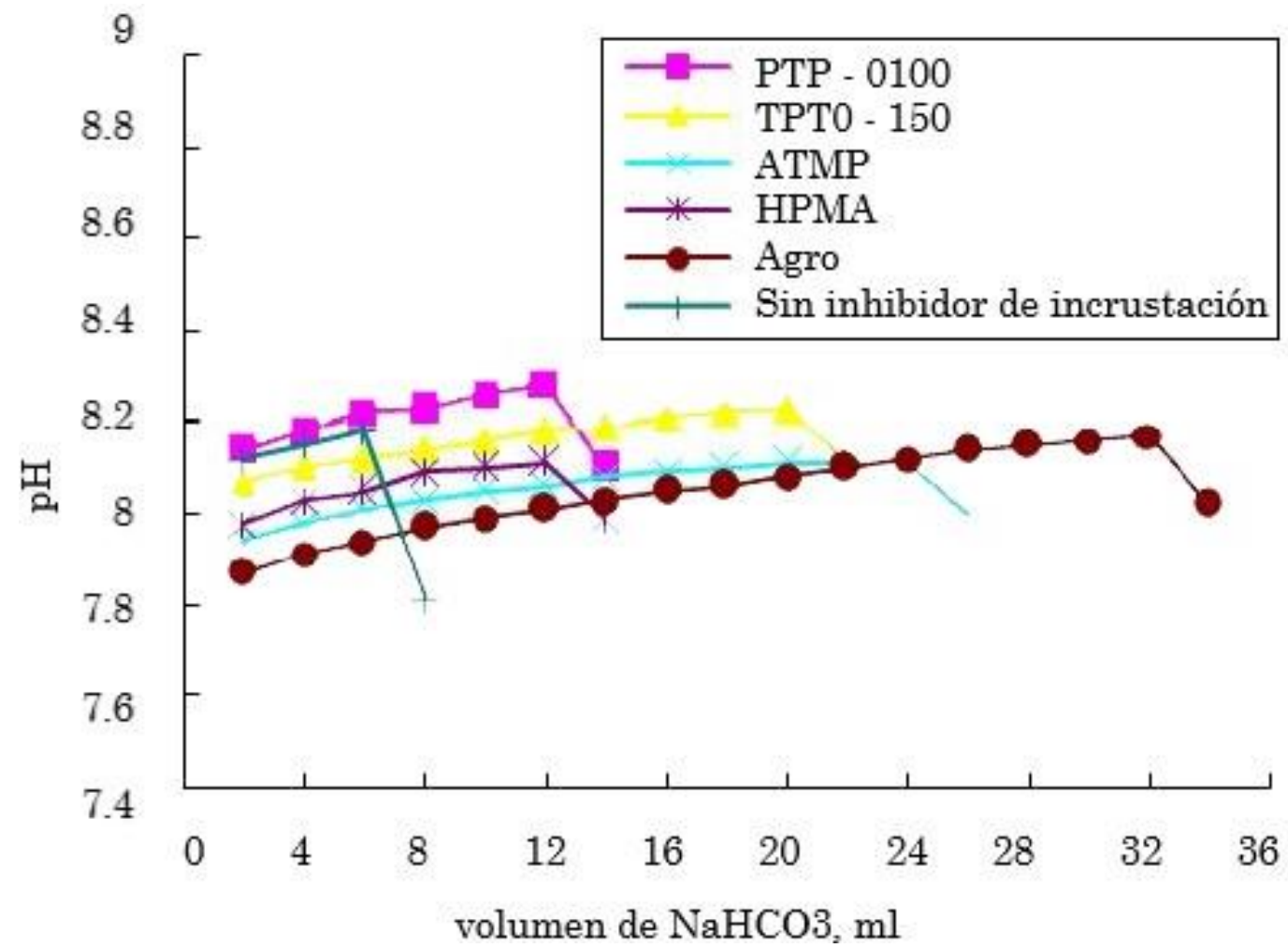

Fig. 5. Comportamiento de $\mathrm{pH}$ en muestra de agua sintética con diferentes inhibidores y sin inhibidor desarrollado por Zhang et al. Fuente: [31]. 
Durante esta etapa Q1 tuvo un comportamiento que consumió $274 \mathrm{~mL}$ de bicarbonato de sodio $\left(\mathrm{NaHCO}_{3}\right)$ hasta que se alcanzó el umbral de precipitación.

En este punto, para todas las muestras de aguas se calculó el índice de supersaturación (S) que permitió determinar cuantitativamente el nivel de incrustación de las mismas al finalizar la prueba, describiendo a este parámetro (S) como la fuerza impulsora que conduce a la cristalización de $\mathrm{CaCO}_{3}$ [54].

Los resultados se encuentran en la Tabla 12 y a partir de allí, el desempeño de los inhibidores se calificó tomando en cuenta el criterio de valoración expuesto en el planteamiento de la metodología.

Tabla 12. Resultados de supersaturación luego de la aplicación del método de medición. Fuente: Autores

\begin{tabular}{|c|c|c|c|c|c|}
\hline Muestra & Inhibidor & Dosis ppm & $\mathrm{S}( \pm 0,60)$ & VIS, \% & Tiempo, min \\
\hline \multirow{10}{*}{$\begin{array}{c}1(3,33 \mathrm{~g} \\
\mathrm{CaCl}_{2}+2,52 \mathrm{~g} \\
\left.\mathrm{NaHCO}_{3}\right)\end{array}$} & \multirow{4}{*}{$\mathrm{Q}_{1}$} & 0 & 194 & 45,47 & 13 \\
\hline & & 55 & 203 & 48,10 & 45 \\
\hline & & 100 & 301 & 71,33 & 86 \\
\hline & & 300 & 373 & 88,39 & 137 \\
\hline & \multirow{3}{*}{$\mathrm{Q}_{2}$} & 55 & 199 & 47,16 & 53 \\
\hline & & 100 & 318 & 75,36 & 83 \\
\hline & & 300 & 422 & 100 & 146 \\
\hline & \multirow{3}{*}{$\mathrm{Q}_{3}$} & 55 & 104 & 24,64 & 18 \\
\hline & & 100 & 130 & 30,81 & 23 \\
\hline & & 300 & 177 & 41,94 & 33 \\
\hline \multirow{10}{*}{$\begin{array}{c}2(6,33 \mathrm{~g} \\
\mathrm{CaCl}_{2}+2,52 \mathrm{~g} \\
\left.\mathrm{NaHCO}_{3}\right)\end{array}$} & & 0 & 239 & 47,90 & 35 \\
\hline & \multirow{3}{*}{$\mathrm{Q}_{1}$} & 55 & 347 & 69,54 & 112 \\
\hline & & 100 & 397 & 79,56 & 139 \\
\hline & & 300 & 499 & 100 & 187 \\
\hline & \multirow{3}{*}{$\mathrm{Q}_{2}$} & 55 & 246 & 49,30 & 102 \\
\hline & & 100 & 294 & 58,92 & 118 \\
\hline & & 300 & 310 & 62,12 & 152 \\
\hline & \multirow{3}{*}{$\mathrm{Q}_{3}$} & 55 & 195 & 39,08 & 32 \\
\hline & & 100 & 197 & 39,48 & 30 \\
\hline & & 300 & 235 & 47,09 & 37 \\
\hline \multirow{10}{*}{$\begin{array}{c}3(2,33 \mathrm{~g} \\
\mathrm{CaCl}_{2}+5,52 \mathrm{~g} \\
\left.\mathrm{NaHCO}_{3}\right)\end{array}$} & & 0 & 24 & 13 & 11 \\
\hline & \multirow{3}{*}{$\mathrm{Q}_{1}$} & 55 & 133 & 77,33 & 122 \\
\hline & & 100 & 154 & 89,53 & 158 \\
\hline & & 300 & 172 & 100 & 172 \\
\hline & \multirow{3}{*}{$\mathrm{Q}_{2}$} & 55 & 119 & 69,19 & 134 \\
\hline & & 100 & 146 & 84,88 & 166 \\
\hline & & 300 & 155 & 90,11 & 160 \\
\hline & \multirow{3}{*}{$\mathrm{Q}_{3}$} & 55 & 29 & 16,84 & 13 \\
\hline & & 100 & 33 & 19,19 & 14 \\
\hline & & 300 & 44 & 25,58 & 17 \\
\hline
\end{tabular}


En la muestra 1 el inhibidor Q2 presentó un índice de supersaturación de 422 a la máxima dosificación, y para las muestras 2 y 3, Q1 fue el que tuvo el mejor desempeño al arrojar índices de supersaturación de 499 y 172, respectivamente, ambos utilizando su máxima dosis. En cada una de las muestras, estos valores fueron los mejores desempeños, por lo tanto, se utilizaron como punto máximo de eficiencia al momento de comparar el comportamiento del resto de las dosificaciones utilizadas.

Partiendo de allí, tanto Q1 como Q2 se consideran inhibidores muy efectivos de acuerdo con el criterio de valoración establecido para esta etapa $\mathrm{y}$, por otra parte, a pesar de que Q3 obtuvo índices de supersaturación inferiores al resto de los químicos, también alcanzó a retrasar el umbral de precipitación, sin embargo, los valores obtenidos de acuerdo con el criterio de valoración lo catalogan como un inhibidor de regular a deficiente.

$\mathrm{Al}$ culminar la aplicación de las distintas etapas que conforman esta metodología, se presenta el desempeño de los químicos evaluados en una matriz evaluativa (Tabla 13) que permitió determinar, a través del puntaje acumulado, el desempeño general de los inhibidores.

De acuerdo con lo presentado en la Tabla 12, Q1 obtuvo un comportamiento similar tanto para la muestra 1 como la muestra 2 , presentando un puntaje que va desde 18 hasta 27 puntos y en la muestra 3 , se observa que el químico presentó el puntaje más alto (27) en sus tres dosis, por lo tanto, de acuerdo con el criterio de valoración establecido en la leyenda anexa, se trata de un antiincrustante catalogado de efectivo a muy efectivo.

Así mismo, el inhibidor Q2 obtuvo un comportamiento similar al químico antes mencionado, presentando sus mejores desempeños para las muestras de agua $1 \mathrm{y}$ 3 , pero exteriorizando deficiencias en la salmuera sintética 2 , lo cual se asocia a la concentración de los reactivos empleados en ella, específicamente a la cantidad de cloruro de calcio que favorece la supersaturación de la solución [55].

A pesar de ello, ambos químicos se consideran eficientes al momento de retrasar la formación de incrustaciones en las muestras evaluadas. Al hablar del inhibidor Q3, es preciso mencionar que este presenta un puntaje que va desde 3 hasta 15 puntos, siendo este último su máximo desempeño en la muestra de agua 3 , describiéndolo como un químico poco efectivo al momento de reducir la formación de incrustaciones minerales.

Este resultado se encuentra asociado al comportamiento del inhibidor en las distintas etapas que conforman la metodología; entre ellos los resultados obtenidos en la etapa de determinación del índice de saturación de Langelier, donde se confirmó el aporte de calcio al sistema.

Sabiendo esto, de los tres antiincrustantes evaluados, Q3 es considerado la última opción de los tres tratamientos químicos estudiados en la presente investigación, a menos que la muestra de agua empleada presente baja proporción de compuestos cálcicos. 
Nueva metodología para la evaluación de productos inhibidores de incrustación basada en modelos existentes

Tabla 13. Matriz evaluativa para determinar efectividad de los inhibidores evaluados con resultados obtenidos Fuente: elaboración propia.

\begin{tabular}{|c|c|c|c|c|c|c|}
\hline & Inhibidor & Dosis & $\begin{array}{c}\text { NACE TM037 - } \\
2007\end{array}$ & $\begin{array}{c}\text { Variación de } \\
\text { tendencia } \\
\text { incrustante }\end{array}$ & $\begin{array}{c}\text { Medición } \\
\text { continua de } \\
\text { pH }\end{array}$ & $\begin{array}{c}\text { Desempeño } \\
\text { general }\end{array}$ \\
\hline \multirow{9}{*}{ 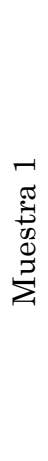 } & \multirow{3}{*}{$\mathrm{Q} 1$} & 55 & 7 & 7 & 4 & 18 \\
\hline & & 100 & 10 & 4 & 7 & 21 \\
\hline & & 300 & 10 & 7 & 10 & 27 \\
\hline & \multirow{3}{*}{ Q2 } & 55 & 10 & 4 & 4 & 18 \\
\hline & & 100 & 10 & 4 & 10 & 24 \\
\hline & & 300 & 10 & 4 & 10 & 24 \\
\hline & \multirow{3}{*}{ Q3 } & 55 & 1 & 1 & 1 & 3 \\
\hline & & 100 & 1 & 1 & 4 & 6 \\
\hline & & 300 & 4 & 1 & 4 & 9 \\
\hline \multirow{9}{*}{ 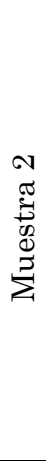 } & \multirow{3}{*}{$\mathrm{Q} 1$} & 55 & 7 & 4 & 7 & 18 \\
\hline & & 100 & 10 & 4 & 10 & 24 \\
\hline & & 300 & 10 & 1 & 10 & 21 \\
\hline & \multirow{3}{*}{ Q2 } & 55 & 1 & 4 & 4 & 9 \\
\hline & & 100 & 7 & 4 & 7 & 18 \\
\hline & & 300 & 4 & 1 & 7 & 12 \\
\hline & \multirow{3}{*}{ Q3 } & 55 & 1 & 1 & 4 & 6 \\
\hline & & 100 & 1 & 1 & 4 & 6 \\
\hline & & 300 & 1 & 1 & 4 & 6 \\
\hline \multirow{9}{*}{ 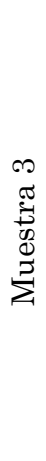 } & \multirow{3}{*}{$\mathrm{Q} 1$} & 55 & 10 & 7 & 10 & 27 \\
\hline & & 100 & 10 & 7 & 10 & 27 \\
\hline & & 300 & 10 & 7 & 10 & 27 \\
\hline & \multirow{3}{*}{$\mathrm{Q} 2$} & 55 & 4 & 10 & 7 & 21 \\
\hline & & 100 & 10 & 7 & 10 & 27 \\
\hline & & 300 & 7 & 7 & 10 & 24 \\
\hline & \multirow{3}{*}{ Q3 } & 55 & 1 & 1 & 1 & 3 \\
\hline & & 100 & 1 & 1 & 1 & 3 \\
\hline & & 300 & 4 & 7 & 4 & 15 \\
\hline
\end{tabular}

\footnotetext{
* Desempeño general

Inhibidor deficiente

Inhibidor regular

Inhibidor efectivo

Inhibidor muy efectivo
}

\section{CONCLUSIONES}

La metodología diseñada permite determinar la eficiencia de productos químicos antiincrustantes tomando en cuenta la variación de propiedades fisicoquímicas de muestras de agua posterior a la dosificación de los mismos, donde la aplicación conjunta de las distintas fases que corresponden a la norma NACE TM 0374-2007, el Índice de Saturación de Langelier y el modelo de medición de $\mathrm{pH}$ continuo desarrollado por Zhang, Wu, Li y Zhao, facilita la selección 
del inhibidor más óptimo según el escenario que se presente. Sabiendo esto, el procedimiento es adaptable tanto a las muestras de agua con tendencia a generar incrustaciones de carbonato de calcio, como a los tratamientos químicos a evaluar $\mathrm{y}$ permite visualizar los resultados obtenidos en cada una de las etapas que la conforman.

$\mathrm{Al}$ evaluar la metodología empleando tres muestras de aguas sintéticas y tres productos inhibidores, con características distintas, el estudio arrojó que la totalidad de las muestras de agua poseían tendencia a formar incrustaciones de carbonato de calcio de acuerdo con el índice de saturación de Langelier (1,302; 1,3241 y $0,8347)$ y con su condición alcalina debido a los valores de $\mathrm{pH}$ obtenidos.

Por otra parte, se observó que el desempeño de los inhibidores químicos evaluados es directamente proporcional a la dosificación aplicada y esta se vio afectada por la concentración salina que presente la muestra, específicamente la concentración de cloruro de calcio, al tratarse de la sal predominante en la formación de $\mathrm{CaCO}_{3}$. Se evidenció que el inhibidor Q3 al estar elaborado de materia prima orgánica que no logró disolverse completamente, presentó inconvenientes al momento de ejercer su función antiincrustante debido a los aportes de sólidos que incrementaban la tendencia incrustante del agua.

Como estudios y planteamientos futuros se espera aplicar la metodología diseñada en muestras de agua de producción y mezclas de ellas, con composiciones y características más complejas para así poder ir adaptando la propuesta presentada en esta investigación, además de aplicarla en otros inhibidores de incrustación, sean de origen orgánico o inorgánico con el fin de ampliar su campo de estudio y permitir la optimización de la misma.

\section{REFERENCIAS}

[1] K. Bello, "Propuesta de programa de estimulación matricial reactiva a pozos productores que presentan merma de producción por incrustaciones en el área Dación del distrito San Tomé," (Trabajo de grado), Universidad de Oriente, Anzoátegui, 2010. URL

[2] R. W. Keatch, "Removal of sulphate scale from surfaces," Application Patent 2314865 A, January 14, 1998. URL

[3] J. R. Becker, Corrosion and Scale Handbook., United States: Penn Well Publishing Company, 1998.

[4] B. Tomaszewska; M. Tyszer, "Assessment of the influence of temperature and pressure on the prediction of the precipitation of minerals during the desalination process," Desalination, vol. 424, pp. 102-109, Dec. 2017.

https://doi.org/10.1016/j.desal.2017.10.003

[5] P. Zhang; D. Shen; G. Ruan; A. T. Kan; M. B. Tomson, "Phosphino-polycarboxylic acid modified inhibitor nanomaterial for oilfield scale control: Synthesis, characterization and migration," Journal of Industrial and Engineering Chemistry, vol. 45, pp. 366374, Jan. 2017. https://doi.org/10.1016/j.jiec.2016.10.004

[6] T. Kumar; S. Vishwanatham; S .S. Kundu, "A laboratory study on pteroyl-L-glutamic acid as a scale prevention inhibitor of calcium carbonate in aqueous solution of synthetic produced water," Journal of Petroleum Science and Engineering, vol. 71, no. 1-2, pp. 1-7, Mar. 2010. https://doi.org/10.1016/j.petrol.2009.11.014

[7] D. Liu, "Research on Performance Evaluation and Anti-scaling Mechanism of Green Scale Inhibitors by Static and Dynamic Methods,"(Tesis Doctoral), Ecole nationale supérieure d'arts et métiers ENSAM, Paris, 2011. URL

[8] E. J. Mackay; I. R. Collins; M. M. Jordan; N. Feasey, "PWRI: scale formation risk assessment and management," in The SPE 5th International Symposium on Oilfield Scale, Jan. 2003, pp. 1-18.

https://doi.org/10.2118/80385-MS

[9] P. Zhang; Y. Liu; N. Zhang; W. Fail Ip; A. T. Kan; M. B. Tomson, "A novel attach-andrelease mineral scale control strategy: Laboratory investigation of retention and release of scale inhibitor on pipe surface," Journal of Industrial and Engineering Chemistry, vol. 70, pp. 462-471, Feb. 2019. https://doi.org/10.1016/j.jiec.2018.11.009

[10] S. Baraka-Lokmane; K. S. Sorbie, "Effect 
Nueva metodología para la evaluación de productos inhibidores de incrustación basada en modelos existentes

of $\mathrm{pH}$ and scale inhibitor concentration on phosphonate-carbonate interaction," Journal of Petroleum Science and Engineering, vol. 70, pp. 10-27, 2010. https://doi.org/10.1016/j.petrol.2009.05.002

[11] A. E. Elkholy; F. El-Taib Heakal; A. M. Rashad; K. Zakaria, "Monte Carlo simulation for guar and xanthan gums as green scale inhibitors," Journal of Petroleum Science \& Engineering, vol. 166, pp. 263-273, Jul. 2018.

https://doi.org/10.1016/j.petrol.2018.03.019

[12] S. P. R. Holt; J. Sanders; K. Rodriguez; M. Vanderhoof, "Biodegrable Alternatives for Scale Control in The SPE 5th International Symposium on Oilfield Scale, Jan. 2009, pp. 1-10.

https://doi.org/10.2118/121723-MS

[13] A Viloria; L. Castillo; J. A. Garcia, M. A. Carrasquero; E. V. Torin, "Process using Aloe for inhibiting scale," United States of America, B2 Patent 8,039,421, octubre 18, 2011. URL

[14] D. Malavé; T. Marín; V. Acevedo; M. Parra, "Extracto de Okra (Abelmoschus esculentus) como aditivo inhibidor de carbonato de calcio en aguas de producción del Campo El Furrial, Estado Monagas, Venezuela," Revista Tecnológica ESPOL $R T E$, vol. 29, no. 2, pp. 1-11, Dec. 2016. URL

[15] A. S. Reyes Sisco; J. A. Ruiz Arenas; L. A. Castillo Campos, "Inhibidor de incrustaciones natural a base del mucílago de la hoja de cayena (Hibiscus rosa sinensis)," Enfoque UTE, vol. 10, no. 2, pp. 63-78, Jun. 2019.

https://doi.org/10.29019/enfoque.v10n2.460

[16] M. F. B. Sousa; F. Signorelli; C. A. Bertran, "Fast evaluation of inhibitors for calcium carbonate scale based on $\mathrm{pH}$ continuous measurements in jar test at high salinity condition," Journal of Petroleum Science and Engineering, vol. 147, pp. 468-473, Nov. 2016. http://dx.doi.org/10.1016/j.petrol.2016.09.0 $\underline{07}$

[17] National Association of Corrosion Engineers (NACE), NACE Standard TM0374-2016: Standard Test Method:Laboratory Screening Tests to Determine the Ability of Scale Inhibitors to Prevent the Precipitation of Calcium Sulfate and Calcium Carbonate from Solution (for Oil and Gas Production Systems). Houston, Texas, U.S.A: NACE, 2016. URL

[18] National Association of Corrosion Engineers (NACE), NACE Standard
TM0197-2019: Laboratory Screening Test to Determine the Ability of Scale Inhibitors to Prevent the Precipitation of Barium Sulfate or Strontium Sulfate, or Both, from Solution (for Oil and Gas Production Systems). URL

[19] D. Liu; W. Dong; F. Li, F. Hui; J. Lédion, "Comparative performance of polyepoxysuccinic acid and polyaspartic acid on scaling inhibition by static and rapid controlled precipitation methods," Desalination, vol. 304, pp. 1-10, Oct. 2012. https://doi.org/10.1016/j.desal.2012.07.032

[20] Q. Wang et al., "Laboratory study on efficiency of three calcium carbonate scale inhibitors in the presence of EOR chemicals," Petroleum, vol. 4, no. 4, pp. 375384, Dec. 2018.

https://doi.org/10.1016/j.petlm.2018.03.003

[21] A. Al Helal; A Soames; S. Iglauer; R. Gubner; A. Barifcani, "Evaluating chemical-scale-inhibitor performance in external magnetic fields using a dynamic scale loop," Journal of Petroleum Science and Engineering, vol. 179, pp. 1063-1077, Aug. 2019.

https://doi.org/10.1016/j.petrol.2019.04.093

[22] O. S. Sanni; O. Bukuaghangin; T. V. K. Charpentier; A. Neville, "Evaluation of laboratory techniques for assessing scale inhibition efficiency," Journal of Petroleum Science and Engineering, vol. 182, Nov. 2019. https://doi.org/10.1016/j.petrol.2019.10634 7

[23] $\bar{R}$ G. M de A. Macedo et al., "Water-soluble carboxymethylchitosan as green scale inhibitor in oil wells," Carbohydrate Polymers, vol. 215, pp. 137-142, Jul. 2019. https://doi.org/10.1016/j.carbpol.2019.03.08 $\underline{2}$

[24] K. D. Demadis; P. Lykoudis, "Chemistry of Organophosphonate Scale Growth lnhibitors: 3. Physicochemical Aspects of 2-Phosphonobutane-1,2,4-Tricarboxylate (PBTC) And Its Effect on CaCO3 Crystal Growth," Bioinorganic Chemistry and Applications, vol. 3, pp. 135-149, 2005. https://doi.org/10.1155/BCA.2005.135

[25] V. Tantayakom; H. S. Fogler; P. Charoensirithavorn; S. Chavadej, "Kinetic Study of Scale Inhibitor Precipitation in Squeeze Treatment," Crystal Growth \& Design, vol. 5, pp. 329-335, Dec. 2004. https://doi.org/10.1021/cg049874d

[26] I. Drela; P. Falewicz; S. Kuczkowska, "New rapid test for evaluation of scale inhibitors," Water Research, vol. 32, no. 10, pp. 3188-3191, Oct. 1998. 
Nueva metodología para la evaluación de productos inhibidores de incrustación basada en modelos existentes

https://doi.org/10.1016/S00431354(98)00066-9

[27] A. P. Morizot; A. Neville, "Using an electrochemical approach for monitoring kinetics of $\mathrm{CaCO} 3$ and $\mathrm{BaSO} 4$ scale formation and inhibition on metal surfaces," SPE Journal, vol. 6, no. 2, pp. 220-223, 2001. https://doi.org/10.2118/71712-PA

[28] J. Xiao; A. T. Kan; M. B. Tomson, "Prediction of $\mathrm{BaSO} 4$ precipitation in the presence and absence of a polymeric inhibitor: phosphino-polycarboxylic acid," Langmuir, vol. 17, no. 15, pp. 4668-4673, Jun. 2001.

https://doi.org/10.1021/la001721e

[29] Y. Liu et al., "An assay method to determine minera lscale inhibitor efficiency in produced water," Journal of Petroleum Science and Engineering, vol. 143, pp. 103-112, Jul. 2016. http://dx.doi.org/10.1016/j.petrol.2016.02.0 24

[30] J. Y. Gal; J-C. Bollinger; H. Tolosa; N. Gache, "Calcium carbonate solubility: a reappraisal of scale formation and inhibition," Talanta, vol. 43, no. 9, pp. 1497-1509, Sep. 1996.

https://doi.org/10.1016/00399140(96)01925-X

[31] X. Ni Zhang; W. Long Wu; D. Mei Li; G. Jin Zhao, "A new evaluation method of scale inhibitors for controlling $\mathrm{CaCO} 3$ scale in reverse osmosis system based on pH measurement," Advanced Materials Research, vol. 356-360, pp. 2146-2152, 2012.

http://dx.doi.org/10.4028/www.scientific.ne t/AMR.356-360.2146

[32] Z. Kiaei; A Haghtalab, "Experimental study of using Ca-DTPMP nanoparticles in inhibition of CaCO3 scaling in a bulk water process," Desalination, vol. 338, pp. 84-92, Apr. 2014.

https://doi.org/10.1016/j.desal.2014.01.027

[33] H-J. Lee; M. A. Halali; T. Baker; S. Sarathy; F. de Lannoy, "A comparative study of RO membrane scale inhibitors in wastewater reclamation: Antiscalants versus pH adjustment," Separation and Purification Technology, vol. 240, Jun. 2020.

https://doi.org/10.1016/j.seppur.2020.1165 $\underline{49}$

[34] E. J. Suárez-Domínguez et al., "Wells Produced Water Conditioning: stabilization for transport and reuse," in Pan American Mature Fields Congress 2015, Veracruz, México, 2015. URL
[35] V. González Dávila; E. Suárez Domínguez, "Stabilized pipe scaling remover and inhibitor compound," United States of America, B2 Patent US 9,085,748 B2, julio 21, 2015. URL

[36] R. Ketrane; B. Saidani; O. Gil; L. Leleyter; F. Baraud, "Efficiency of five scale inhibitors on calcium carbonate precipitation from hard water: effect of temperature and concentration," Desalination, vol. 249, no. 3, pp. 13971404, Dec. 2009.

http://dx.doi.org/10.1016/j.desal.2009.06.013

[37] H. Semine Ras; S. Ghizellaoui, "Determination of Anti-Scale Effect of Hard Water by Test of Electrodeposition," Procedia Engineering, vol. 33, pp. 357-365, 2012.

http://dx.doi.org/10.1016/j.proeng.2012.01.1 $\underline{215}$

[38] W. F. Langelier, "Chemical equilibria in water treatment," Journal of the American Water Works Association, vol. 38, no. 2, pp. 169-178, Feb. 1946. URL

[39] National Fire Protection Association (NFPA), NFPA 704: Standard System for the Identification of the Hazards of Materials for Emergency Response. Massachusetts, U.S.A.: NFPA, 2007. https://doi.org/10.13140/rg.2.2.32358.01608

[40] The California Water Boards. (2020) Water Resources Control Board. en línea: URL

[41] American Public Health Association, APHA 4500-H-+ B : Ph Electrometric Metghod. Satandar Methods for the examination of water and waterwaste. 18th ed. USA: APHA, 1992. URL

[42] Comisión Venezolana de Normas Industriales, COVENIN 2461-87 Aguas naturales, industriales y residuales. Determinación de sólidos, Primera ed. Caracas, Venezuela: Fondonorma, 1987. URL

[43] American Public Health Association, APHA 2510: Standard Methods for the Examination of Water and Waterwaste: Conductivity, 18th ed. Washington, D.C., U.S.A.: APHA, 1992.. URL

[44] American Public Health Association, APHA 2320 A: Standard Methods for the Examination of Water and Waterwaste: Alkalinity, 18th ed. Washington, D.C., U.S.A: APHA, 1992. URL

[45] American Public Health Association, APHA 3500 CA: Standard Methods for the Examination of Water and Waterwaste: Calcium, 18th ed. Washington, D.C., U.S.A.: APHA, 1992. URL

[46] American Public Health Association, APHA 4500 CIA: Standard Methods for the 
Nueva metodología para la evaluación de productos inhibidores de incrustación basada en modelos existentes

Examination of Water and Waterwaste: Chlorine, 18th ed. Washington, D.C., U.S.A.: APHA, 1992. URL

[47] M. Crabtree; D. Eslinger; P. Fletcher; M. Millet; A. Johnson; G. King, "La lucha contra las incrustaciones - remoción y prevención," Oilfield Review Magazine, pp. 30-49, 1999. URL

[48] J. Martín Porras; P. López Guerrero; C. Álvarez Fernandez; A. Fernandez Uría; M. V. Gimeno, Calidad y contaminación de aguas subterráneas en España. España: IGME, 1985. URL

[49] N. F. Gray, Water Technology: An Introduction for Environmental Scientists and Engineers, Second edition ed. U.S.A.: Butterworth-Heinemann, 2005. URL

[50] Environmental Protection Agency (EPA), Guidelines Establishing Test Procedures for the Analysis of Pollutants under the clean Water Act; Analysis and Sampling Procedures. $\quad$ U.S.A.: Environmental Protection Agency, 2013. URL

[51] S. I. Armendáriz et al., "Estudio de la precipitación de carbonato de calcio en un destilador solar experimental," Revista Internacional de Contaminación Ambiental, vol. 21, no. 1, pp. 5-15, 2005. URL

[52] Z. Quan; Y. Chang Chen; X. Rong Wang; C. Shi; Y. J. Liu; C. Fang Ma "Experimental study on scale inhibition performance of a green scale inhibitor polyaspartic acid," Science in China Series B: Chemistry, vol. 51, pp. 695-699, Jun. 2008. https://doi.org/10.1007/s11426-008-0063-y

[53] G. Suo; l. Xie; S- Xu; L. Feng; T. Dong; X. Shao, "Study on inhibitors' performance under the condition of high concentration ratio in MED system," Desalination, vol.
437, pp. 100-107, Jul. 2018. https://doi.org/10.1016/j.desal.2018.02.019

[54] J. W. Mullin, Crystallization, 4th ed. Woburn: Butterworth-Heinemann, 2001. https://doi.org/10.1016/B978-0-7506-48332.X5000-1

[55] D. Konopacka-Łyskawa; B. Koscielska; J. Karczewski; A. Gołabiewska, "The influence of ammonia and selected amines on the characteristics of calcium carbonate precipitated from calcium chloride solutions via carbonation," Materials Chemistry and Physics, vol. 193, pp. 13-18, Jun. 2017. http://dx.doi.org/10.1016/j.matchemphys.201 $\underline{7.01 .060}$

\section{CONTRIBUCIÓN DE LOS AUTORES}

(D) 1 Contribución: desarrolladora de la investigación en cuanto a la realización de pruebas experimentales, además de la redacción inicial del manuscrito presentado y las correcciones del mismo.

(D) 2 Contribución: desarrollador de la investigación en cuanto a la realización de pruebas experimentales y encargado de las correcciones preliminares del manuscrito.

(D) 3 Contribución: autor del tema a desarrollar y de la conceptualización y seguimiento de la investigación, encargado de la redacción del manuscrito presentado y la adaptación y correcciones del mismo. 